\title{
Physical and transcriptional map of the hereditary inclusion body myopathy locus on chromosome 9p12-p13
}

\author{
Iris Eisenberg ${ }^{1}$, Hagit Hochner ${ }^{1}$, Moshe Shemesh ${ }^{1}$, Tatjana Levi ${ }^{2}$, Tamara Potikha ${ }^{1}$, \\ Menachem Sadeh ${ }^{3}$, Zohar Argov ${ }^{4}$, Cynthia L Jackson ${ }^{5}$ and Stella Mitrani-Rosenbaum*,1
}

\begin{abstract}
${ }^{1}$ The Unit for Development of Molecular Biology and Genetic Engineering, Hadassah Hospital, The Hebrew University-Hadassah Medical School, Jerusalem 91240, Israel; ${ }^{2}$ Department of Genetics, Harvard Medical School, Boston, Massachussets 02115, USA; ${ }^{3}$ Department of Neurology, Wolfson Hospital, Holon, Israel; ${ }^{4}$ Department of Neurology, Hadassah Hospital, The Hebrew University-Hadassah Medical School, Jerusalem 91240, Israel; ${ }^{5}$ Department of Pathology, Rhode Island Hospital and Brown University, Providence, Rhode Island 02192, USA
\end{abstract}

Hereditary inclusion body myopathy (HIBM) is a group of neuromuscular disorders characterised by adultonset, slowly progressive distal and proximal muscle weakness and typical muscle pathology. Previously, we have mapped the gene responsible for a recessive form of HIBM to chromosome $9 p 1$ and narrowed the interval to one single YAC clone of $1 \mathrm{Mb}$ in size. As a further step towards the identification of the HIBM gene, we have constructed a detailed physical and transcriptional map of this region. A high resolution BAC contig that includes the HIBM critical region, flanked by marker 327GT4 and D9S1859, was constructed. This contig allowed the precise localisation of 25 genes and ESTs to the proximal region of chromosome 9. The expression pattern of those mapped genes and ESTs was established by Northern blot analysis. In the process of refining the HIBM interval, 13 new polymorphic markers were identified, of which 11 are CA-repeats, and two are single nucleotide polymorphisms. Certainly, this map provides an important integration of physical and transcriptional information corresponding to chromosome 9p12-p13, which is expected to facilitate the cloning and identification not only of the HIBM gene, but also other disease genes which map to this region. European Journal of Human Genetics (2001) 9, 501-509.

Keywords: chromosome 9; IBM2; physical and transcript map; BAC contig; polymorphisms; neuromuscular diseases.

\section{Introduction}

Hereditary inclusion body myopathy (HIBM) (OMIM 600737 ) is a unique group of neuromuscular disorders characterised by adult onset, slowly progressive distal and proximal muscle weakness and a typical muscle pathology including rimmed vacuoles and filamentous inclusions. ${ }^{1-3} \mathrm{~A}$ form of HIBM found in Jews of Persian descent affects most leg muscles except the quadriceps. Our previous studies

${ }^{*}$ Correspondence: S Mitrani-Rosenbaum, The Unit for Development of Molecular Biology and Genetic Engineering, Hadassah Hospital-Mount Scopus, Jerusalem 91240, Israel. Tel: 972-2-5844724; fax: 972-2-5819134 E-mail: stella@yam-suff.huji.ac.il

Received 2 January 2001; revised 15 March 2001; accepted 6 April 2001 localised the gene causing this recessive form of HIBM in Persian Jews within a genomic interval of approximately $1 \mathrm{Mb}$ covered by a single YAC, on chromosome $9 \mathrm{p} 1 .^{4-7}$ Subsequently Christodoulou et $a l^{8}$ have reported the same locus involved in quadriceps sparing HIBM in additional Jewish patients from Persian origin, and in other ethnic groups, and very recently Mirabella et $a l^{9}$ have described an Italian family with autosomal recessive quadriceps-sparing inclusion-body myopathy also linked to this locus. Ikeuchi et $a l^{10}$ have also reported that a distal myopathy with rimmed vacuoles observed in Japanese patients maps to the same locus. This disorder is very similar to HIBM in Persian Jews: onset in early adulthood with drop foot; biopsies show rimmed vacuoles and inclusions of the same nature. The 
main difference consists in the progression which eventually involves the quadriceps within 10 years of onset, resulting in early loss of ambulation of the Japanese patients. Today it is agreed that very likely these conditions are allelic forms of the same disorder. Many previous studies have shown that the short arm of chromosome 9 and in particular the region 9p12-p13 plays an important role in a large number of common diseases, such as acute lymphoblastic leukaemia, ${ }^{11}$ non-Hodgkin's lymphoma, ${ }^{12}$ lung cancer, ${ }^{13}$ hepatocellular carcinoma (HCC), ${ }^{14}$ as well as in rare diseases, such as arthrogryposis multiplex congenita, distal, type 1 (AMCD1), ${ }^{15}$ acromesomeric dysplasia (AMDM), ${ }^{16}$ cartilage hair hypoplasia $(\mathrm{CHH}),{ }^{17}$ and also in a form of distal hereditary motor neuropathy (HMN) found in Jerash. ${ }^{18}$ At present, the availability of a precise physical map of chromosome 9p12-p13 is very restricted, and very few mapped genes have been identified in this region. Therefore, the construction of a contiguous, high-resolution physical and transcriptional map in this defined genomic interval will certainly contribute to the positional cloning of various disease causing genes. In our attempt to identify the HIBM gene, we have established a complete BAC contig of the chromosomal region 9p12-p13, across the $\beta$-tropomyosin gene and the 3 ' end of the PAX5 gene. This contig has allowed the high resolution physical and transcriptional mapping of several genes, expressed sequence tags, and various additional markers in the region. We have precisely positioned 25 transcripts and analysed their expression pattern. Thirteen new polymorphic sites were identified in this study and fine recombination analysis with these markers allowed the restriction of the HIBM critical region between markers $327 G T 4$ and D9S1859, an interval of about $700 \mathrm{~kb}$. Among the genes and ESTs located between these flanking markers, 12 prevail as potential candidate genes for HIBM.

\section{Materials and methods}

\section{Construction of a high resolution $B A C$ contig}

BAC clones were identified by PCR screening of human BAC libraries (Down to the well ${ }^{(i x}$-GenomeSystems Inc; CITB library, Release IV - Research Genetics Inc), according to the manufacturers' instructions, or from the released data of the Sanger Center (RPCI-11 library-BACPAC Resources). BAC DNA from single clones was prepared using the QIAGEN Plasmid Maxi kit. The BAC DNA ends were directly sequenced using primers T7 and Sp6, on an ABI Prism 377 automated sequencer. All end sequences were analysed for repetitive elements using the RepeatMasker web server, and subsequently used as STS to screen the BAC libraries again. This process was repeated until a complete BAC contig was established. To confirm the precision of the contig constructed, the content of each clone in the contig was assessed twice by high stringent hybridisation of known markers and by 40 novel STS markers derived from the BACs ends.

\section{Identification of novel polymorphic markers}

BAC clones were partially restricted with Sau3A (Boehringer Mannheim) and shotgun cloned into BamHI linearised pSPL3 vector (Gibco-BRL). Plated colonies were transfered to nylon membranes (OSMONICS) and hybridised with a Poly(dA-dC).Poly(dG-dT) probe (Amersham-Pharmacia Biotech). Plasmid inserts from positive colonies were sequenced and PCR amplification primers flanking the repeated region were designed. By searching the Sanger Center recently released BAC sequences, we have been able to identify additional repeat sequences. These sequence data were produced by the Human Chromosome 9 Sequencing Group at the Sanger Center and can be obtained from ftp:// ftp.sanger.ac.uk/pub/human/sequences/Chr_9/. Each marker was tested on several unrelated individuals to determine its polymorphic status.

Genotyping with the newly identified markers was performed using amplification with radioactive labelled PCR primers, and denaturing polyacrylamide gel electrophoresis as previously described. ${ }^{4}$

\section{Transcript map}

All genes and ESTs previously mapped to the region between D9S163 and D9S50 were selected for further localisation based on the following maps and databases: The human transcript map ${ }^{19}$ (http://www.ncbi.nlm.nih.gov/genemap/); The Sanger Center-Map 9ctg3 (http://www.sanger.ac.uk/ HGP/Chr9/); The Stanford RH map (http://shgc-www.stanford.edu/Mapping/rh/search.html); The NCBI Unigene database (http://www.ncbi.nlm.nih.gov/UniGene/ index.html); The Genexpress IMAGE knowledge Base of the Human Muscle Transcriptome: http://idefix.upr420.vjf.cnrs.fr/IMAGE/Page_unique/welcome_muscles.html.

All the selected ESTs were precisely mapped within the HIBM contig by high stringency hybridisation. In addition, all new genomic sequences derived from the BAC clone insert ends and the shotgun sequences were systematically compared with EST databases using the BLASTN 2.0 software. ${ }^{20}$ Sequences found to match additional ESTs were also tested against the BAC contig by PCR amplification and hybridisation. The resulting integrated map is shown in Figure 1.

\section{Northern blot analysis}

To assess tissue distribution and sizes the transcripts mapped inside and in the vicinity of the candidate interval, multiple tissue Northern (MTN, Clontech) blots were used. Hybridisation in ExpressHyb solution (Clontech) and washes were done as recommended by the manufacturer. The blots contain polyA RNA from the following tissues: heart, brain, placenta, lung, liver, skeletal muscle, kidney and pancreas. Hybridisation probes were prepared by $\left[\alpha^{32} \mathrm{P}\right] \mathrm{dCTP}$ labeled PCR generated DNA fragments. Table 1 summarises all primers used for these ESTs amplifications. 


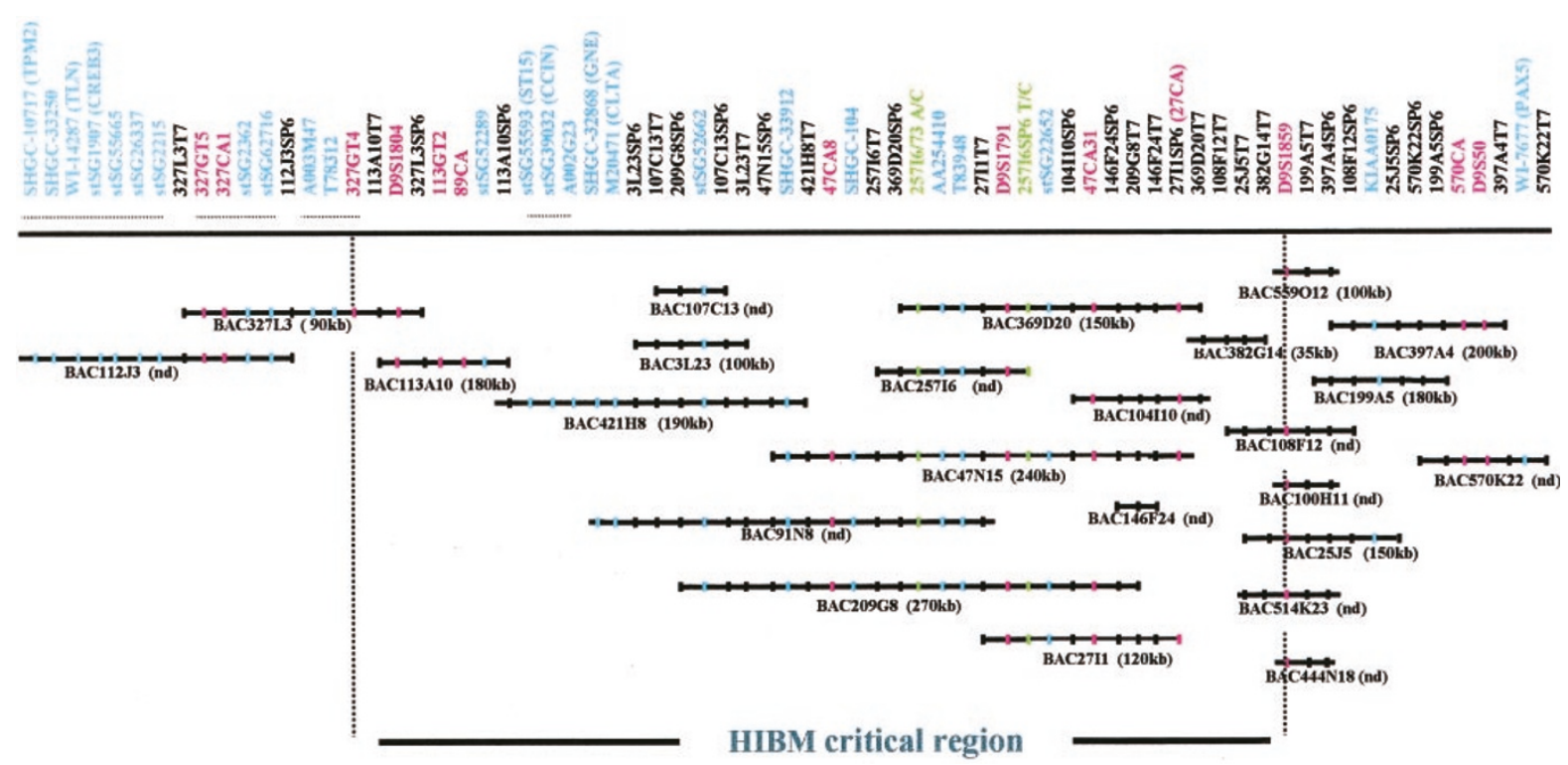

Figure 1 Physical and transcript map of the HIBM region on chromosome 9p12-p13. BAC clones are represented by horizontal lines. The name of each clone and its estimated size is shown below (nd=not done). Filled squares indicate the presence of markers in the clones. Black squares indicate novel STSs generated from BAC ends; blue squares show ESTs and genes; polymorphic markers are in red and the two novel SNPs are indicated in green. 89CA is a polymorphic marker described by Vakkilainen et al. $^{34}$ Non polymorphic microsatellites are not shown. The precise location of BAC559O12, BAC100H11, BAC514K23 and BAC444N18 ends was not tested. The internal order in the underlined clusters is not definitive. Note that the map is not to scale.

Electronic database information

Data were taken from the following websites:

(1) Whitehead Institute for Biomedical Research/MIT Center for Genome Research (WICGR): http://www. genome.wi.mit.edu

(2) The Genome database: http://www.gdb.org

(3) Unigene database chromosome 9: http://www. ncbi.nlm.nih.gov/UniGene/Hs_DATA/ChromLists/ Chr9.html

(4) Blast search: http://www.ncbi.nlm.nih.gov/BLAST

(5) The human transcript map (GeneMap99): http:// www.ncbi.nlm.nih.gov/genemap

(6) FASTA searches: http://www2.igh.cnrs.fr/bin/fastaguess.cgi

(7) The Sanger Center database: http://www.sanger.ac.uk

(8) Summary map of chromosome 9: http://cedar.genetics. soton.ac.uk/pub/chrom9/map.html

(9) The Stanford RH map: http:// shgc-www.stanford.edu/ Mapping/rh/search.html

(10) The cooperative human linkage center: http://cgap. nci.nih.gov/CHLC

(11) The Genexpress IMAGE knowledge Base of the Human Muscle Transcriptome: http://idefix.upr420.vjf.cnrs.fr/ IMAGE/Page_unique/welcome_muscles.html

(12) The RepeatMasker web server: http://ftp.genome. Washington.edu/cgi_bin/Repeat Masker.
Pulsed-field gel electrophoresis

NotI (New England Biolabs, Inc.) digested BAC DNAs were subjected to $1 \%$ agarose gels (Seakem, FMC BioProducts), and separated by pulsed-field gel electrophoresis with a CHEF DRII system(BioRad), in $0.5 \% \mathrm{TBE}$ at $14^{\circ} \mathrm{C}$ for $16 \mathrm{~h}, 6.0 \mathrm{~V} / \mathrm{cm}$, with an initial and final switch time of $30 \mathrm{~s}$. Following electrophoresis, the gel was stained with ethidium bromide for visualisation of the digested fragments.

\section{Results \\ Construction of BAC contig}

As a further resource for cloning the HIBM disease gene, a genomic BAC contig flanked by the $\beta$-tropomyosin gene and the PAX5 gene, encompassing the HIBM critical region, in the chromosome 9p12-p13 region, was generated. BAC libraries were initially screened using three anchor markers D9S1804, D9S1791 and D9S50. Ten BAC clones were identified as positive for these markers (Figure 1). The end sequences of the identified clones were determined by direct sequencing of BAC DNA using primers T7 and Sp6, complementary to the BAC vector sequence. The end sequences were used to develop STS markers for screening BAC libraries again. This process was repeated until all gaps were closed and a complete BAC contig was achieved (Figure 1). Overlap between clones was identified by amplification 
Table 1 Primer sequences used in the analysis of the transcripts mapped within chromosome 9p12-p13 BAC contig

\begin{tabular}{|c|c|c|c|}
\hline EST name & Forward Primer $\left(5^{\prime}, 3^{\prime}\right)$ & Reverse Primer $\left(5^{\prime}, 3^{\prime}\right)$ & Size $(b p)$ \\
\hline stSG55665 & TGT CCA GAG GCT GAC GTA G & TTA CAC AGA GAC CAG CAG GC & 115 \\
\hline stSG26337 & GCC GAA TGA TAC AGC CCT TA & AAA GAA GGA GCT GTC CTG AGG & 138 \\
\hline SHGC-10717 & GGA GCA GAA ATT GCC AAC AT & GGG TGG AAG GGG ATA GGT AA & 116 \\
\hline SHGC-33250 & TAC AAT TAG GAG CAA GTA AGA AGC G & TAC ACC AGG AAC TGA AAT ATC ACT G & 133 \\
\hline Wl-14287 & ATC ATT TTT ATT GGA GCA TGA CC & GTT CCA GCC AGT CCC CAC & 132 \\
\hline stSG1907 & TTG TAC CCA TGT GTC TGT CAC & GGG TGA CCT AGG CAG CAT TA & 131 \\
\hline stSG2215 & GTG ATG AAA ATC TCA GGG AAG G & CTC TGA AGT GGG AGA CGG AG & 183 \\
\hline stSG2362 & AGG CAC AGA CAT TCC CAA AC & CTG GAA GCT GCA GTC TAC ACC & 147 \\
\hline stSG62716 & AGT CCC AGC TCC ATC CAT C & CAG CAT CTT TCC AGA GGA GG & 159 \\
\hline $\mathrm{A} 003 \mathrm{M} 47$ & GAA CAG GAA TAA AGA AAT CTG & GAT TCA TCC AAG CTT AAA & 124 \\
\hline T78312 & ССT TAT GTC CAA ATC TTT GTG C & CCA TGT GGT GAA GTA GGC AG & 192 \\
\hline stSG52289 & GCA AAG ATG GAG ACG GIT GT & AAG GCA GAT CTG TGC TCT CTG & 122 \\
\hline stSG55593 & TCC AGT ATA TTG CAT TAA AGA CAT T & TCT GAA ATA CAT AGG GAT ATG CGT A & 147 \\
\hline stSG39032 & CTG CCC CTT GGA TGT GTC & TCC AGC AAG AAG GCA TTT & 132 \\
\hline A002G23 & GCA TAG CTT GAG ACT GGT GA & GCA ACT TTA CAA GGT CCA TGT GG & 228 \\
\hline M20471 & GAA GCT CTT CAC AGT CAT TGG & ACA GGT TGA GGC TAC TCT TGG & 189 \\
\hline SHGC-32868 & AGA AAT CAT GTG CAT ATA TCT TTC & TTC ACA CTA ACC CAC TAG GAA GG & 139 \\
\hline stSG52662 & AAC CTC CCС TTA AAA AGG CA & ACC TAA ACT GAG AGC AAG CTG G & 159 \\
\hline SHGC-33912 & AGA CTC CTA GGT GTG GAC CAT TGG & GAA TTA AGT TTG GGA TGT TIT CCC & 182 \\
\hline SHGC-104 & TGG TTA CCT CAT TTT GCC GTT TC & TCA AGC CTT CAA ATG ATT TGG TTA C & 164 \\
\hline AA254410 & ССT AAC AAG GTG ATT TGT GAA AG & CTG GAA GAA AGC TTT GAA GTG AGC & 118 \\
\hline T83948 & AAT ATC ACG GTT CTG TCT TT & AAA ATC CAA AAT GCT TCT GGT CCC & 420 \\
\hline stSG22652 & TGA AGA AGA GGG GTC ATG TAC A & CAG GAG GCC AAG AAA GGA G & 162 \\
\hline KIAA0175 & GGA TGA GTG TGG GTG TGA TAC & TGA CAG ATG GGC TTG ATT TAG & 173 \\
\hline Wl-7677 & CAG AAC AGC CAG GTA GAG CC & ATG ATC CTC TCA CAG CCC C & 329 \\
\hline
\end{tabular}

and hybridisation of the same STS markers in multiple clones.

Four clones from the RPCI-11 library - BAC112J3; BAC327L3; BAC113A10; BAC421H8 - were mapped in this locus by the Sanger Center; we confirmed their location in this contig by hybridization with the relevant markers as illustrated in Figure 1. A total of 23 BAC clones were identified, (four from the RPCI-11 library, as mentioned above, and 19 by library screening: nine from Genome Systems - BAC107C13; BAC3L23; BAC91N8; BAC104I10; BAC27I1; BAC146F24; BAC199A5; BAC25J5; BAC108F12, and the remaining 10 from Research Genetics) and 40 novel sequence tagged sites markers were developed from the end sequences of these BAC clones (Figure 1; primer sequences are available upon request). These STS were used to construct a high resolution physical map for the region from $\beta$ tropomyosin gene to the $3^{\prime}$ end of PAX5 gene (WI-7677) (Figure 1), creating a tiling path of the candidate interval. The sizes of inserts were determined by PFGE after NotI digestion, and according to sequence data. The size of the contig was estimated based on the sizes of individual clones and potential overlap between clones.

\section{Identification of novel polymorphic markers}

In the process of constructing this detailed physical map we have identified several new polymorphic markers within the established contig. Eleven novel microsatellites and two single nucleotide polymorphisms were detected (Table 2 ). All repeat markers except 444CA, 327GT6 and 327CA3, were found to be polymorphic in the studied populations. Markers
444CA and 27CA were derived from the Sp6 end sequences of BAC444N18 and 27I1, respectively. 570CA was identified through sequencing a random plasmid subclone derived from BAC570K22, and 47CA31 and 47CA8 were identified in BAC47N15 by hybridisation with a Poly (dA-dC).Poly(dG-dT) probe, as described in Materials and methods. All the remaining repeats $(113 G T 2,327 G T 6,327 G T 4,327 C A 1$, 327GT5) were defined from the published sequences of BACs 113A10 and 327L3 (Accession numbers, AL138834 and AL135841).

The precise location of these markers was determined by STS content mapping in all BAC clones within the contig (Figure 1). Additionally, two single nucleotide polymorphisms were found in BAC257I6. 257Sp6 C/T polymorphism was found in the sequence derived from the Sp6 end of BAC 257 I6 and the second polymorphism, a base substitution $C$ to A, was identified while sequencing a subclone of BAC257I6. Both polymorphisms were found to create a restriction site for MseI (T’TAA).

\section{Transcript map}

In an attempt to obtain a more complete transcription map of the genomic contig generated, we searched several databases for ESTs and genes that potentially map to chromosome 9p12-p13, between the $\beta$-tropomyosin gene and marker D9S50. More than 50 transcripts were selected for this fine mapping study.

Twenty-five different transcripts were unambiguously positioned and ordered in the transcription map we constructed. Most of those were hybridised on MTN blots, 
Table 2 New polymorphic markers identified within chromosome 9p12-p13 BAC contig

\begin{tabular}{|c|c|c|c|c|}
\hline Locus name & Parent clone & Forward primer $\left(5^{\prime}, 3^{\prime}\right)$ & Reverse primer $\left(5^{\prime}, 3^{\prime}\right)$ & Size (bp) \\
\hline $327 C A 1$ & $327 L 3$ & CAG ATG AAC TTA TTC CAG G & СTA CCT AGT GCT ATT ACT AG & 118 \\
\hline 327GT4 & $327 \mathrm{~L} 3$ & TAT GTC TTC ATT TGG GTG & CTC ATA ACC TAG TTC TCT GC & 152 \\
\hline 113GT2 & $113 \mathrm{~A} 10$ & GTT CTT GAG AGA AAC AGT AC & CTT GTC AGC CTC CAT AAT CAG & 120 \\
\hline 47CA8 & $47 \mathrm{~N} 15$ & ССT GAT GGT TAA TGA TGT CAA GC & GTG GCT TGT ATC TAT AGT CC & 220 \\
\hline 47CA31 & $47 \mathrm{~N} 15$ & СCT TAT AGA ACC TGT GTG AG & GGA TGT GAT CAG AAA ATT CC & 215 \\
\hline \multicolumn{5}{|c|}{ Single nucleotide polymorphisms } \\
\hline 257SP6 T/C & 25716 & GAT CTC AAA GGG TAG TAA CAT CTC & TGC ACG CTT AGA GTT ATG CAA AAG & 270 \\
\hline $257 / 73 \mathrm{~A} / \mathrm{C}$ & 25716 & AAC ATA TGA AAG CAC AAT TAC ATA & ATA TTT TTा CAG TTG TGT TTA TAT & 270 \\
\hline
\end{tabular}

as described in Materials and methods. Negative results were checked twice. Additional expression data was compiled from various databases. Table 3 shows a summary of all the expression data accumulated for those ESTs both from database information and from our Northern blotting results (all ESTs were assayed by Northern blot except stSG55665, stSG26337, stSG2362, stSG62716, stSG52289, T83948 and WI-7677). Figure 1 indicates the precise location of these transcripts in the genomic contig.

Among those, 17 transcripts are ESTs expressed in various tissues, with low or no homology to known genes, while the remaining eight are ESTs derived from genes with known function.

$\beta$-tropomyosin (TPM2), an abundant skeletal muscle protein of $284 \mathrm{aa},{ }^{21,22}$ talin (TLN), a high molecular weight cytoskeletal protein which plays a key role in the assembly of actin filaments and in the spreading and migration of various cell types, ${ }^{23,24}$ luman RNA (CREB3), a cAMP response element (CRE)-binding protein/activating transcription factor 1 protein of the basic leucine zipper superfamily, ${ }^{25}$ ST15, which encodes a membrane anchored glycoprotein of about $110 \mathrm{kDa}$ which plays a role in linking oncogenic signals to tumour invasion and metastasis, ${ }^{26}$ UDP-N-acetylglucosamine2-epimerase/N-acetylmannosamine kinase (GNE), a bifunctional enzyme, which regulates and initiates biosynthesis of $\mathrm{N}$-acetylneuraminic acid (NeuAc), ${ }^{27}$ calicin (CCIN), a polypeptide of 588 amino acids encoded by a $2.2 \mathrm{~kb}$ mRNA, expressed in testis as morphogenic cytoskeletal element in spermiogenic differentiation, ${ }^{28}$ clathrin light polypeptide A (CLTA), a regulatory element in clathrin function which is a main structural component of the lattice cover in the cytoplasm face of the coated pits and coated vesicles, $^{29,30}$ paired box homeotic gene 5 (PAX5), a 391 amino acids protein that plays an important role in B-cell differentiation as well as in neural development and spermatogenesis. $^{31}$

\section{Refinement of the HIBM candidate region}

Previously we restricted the HIBM disease interval between D9S1791 and D9S50, based on recombination events and linkage disequilibrium. ${ }^{7}$ The distal boundary, D9S1791, was defined by a recombination event observed in unaffected individual F34 II-5. ${ }^{7}$ However, very recently, the clinical status of this individual has dramatically changed from unaffected to affected, and therefore his haplotype does not reflect a recombination event, but rather his affected status. Based on these results, D9S1791 cannot be considered as the distal boundary of the HIBM interval. Moreover, haplotype analysis in other families with additional markers allowed us to relocate the HIBM candidate interval between $327 G T 4$ on the telomeric side, and D9S1859 on the centromeric side, based on recombination events observed in affected individuals solely (Figure 2): the recombinant chromosome found in affected individual F10 III-4 defined the distal boundary as $327 G T 4$ and the recombination event observed in individual F5 III-4 placed the proximal boundary as marker D9S1859.

Based on the newly established BAC contig, we conclude that the HIBM critical interval, between markers 327GT4 and D9S1859 covers a region of approximately $700 \mathrm{~kb}$ of DNA.

\section{Current positional candidates}

The BAC physical map allowed us to verify and refine the location of 25 genes and ESTs mapping to chromosome 9p12p13 (Table 3, Figure 1). As a result we have been able to exclude a large number of genes and ESTs as potential HIBM candidate genes. PAX5 and $\beta$-tropomyosin were already excluded in our previous study, ${ }^{7}$ stSG2215, CREB3, SHGC33250, TLN, A003M47, T78312, stSG26337, stSG55665, stSG2362 and stSG62716 were excluded based on the recombination observed with 327 GT4. KIAA0175 transcript was excluded due to recombination event with D9S1859. The 12 remaining transcripts (Table 3 ) located between these two flanking markers can be considered as candidate disease causing genes in HIBM.

Among those, eight are ESTs with no similarity to any known gene, and four (ST15, CCIN, CLTA and GNE) are genes with a known function. CCIN is expressed only in testing. The other three genes are expressed in a variety of tissues, including skeletal muscle, the affected tissue in HIBM. Clathrin light chain A (CLTA) is a regulatory element 
Table 3 Transcripts assigned to chromosome 9p12-p13 BAC contig

\begin{tabular}{|c|c|c|c|c|}
\hline $\begin{array}{l}\text { EST } \\
\text { name }\end{array}$ & $\begin{array}{l}\text { GenBank } \\
\text { Acc. No. }\end{array}$ & Gene/similarity & Known function & Expression data \\
\hline stSG55665 & R45884 & Similarity to KIAA0258 protein & & $\begin{array}{l}\text { Blood, brain, CNS, colon, kidney, lung, ovary, } \\
\text { parathyroid, prostate, uterus }\end{array}$ \\
\hline stSG26337 & H65042 & No similarity & & Blood, lung \\
\hline SHGC-10717 & $\mathrm{X} 06825$ & Tropomyosin beta chain (TPM2) & Cytoskeletal protein & $\begin{array}{l}\text { Blood, bone, brain, colon, skeletal muscle, } \\
\text { heart, lung, ovary, pancreas, prostate, kidney, } \\
\text { spleen, uterus, stomach, thymus }\end{array}$ \\
\hline SHGC-33250 & G27778 & $\begin{array}{l}\text { Low similarity to protein phosphatase } \\
\text { PP2A, regulatory subunit (PR130) }\end{array}$ & & Lung \\
\hline WI-14287 & AF177198 & Talin (TLN) & $\begin{array}{l}\text { Cytoskeletal protein and } \\
\text { putative tumor suppressor }\end{array}$ & $\begin{array}{l}\text { Bone, brain, lung, foreskin, ovary, stomach, } \\
\text { uterus, blood, colon, skeletal muscle, heart, } \\
\text { placenta, liver, kidney, pancreas }\end{array}$ \\
\hline stSG1907 & AF009368 & Luman (CREB3) & Transcription factor LZIP & $\begin{array}{l}\text { Blood, bone, brain, breast, CNS, colon, heart, } \\
\text { kidney, lung, lymph, skeletal muscle, ovary, } \\
\text { pancreas, parathyroid, placenta, prostate, } \\
\text { testis, uterus, liver }\end{array}$ \\
\hline stSG2215 & R37397 & No similarity & & $\begin{array}{l}\text { Brain, heart, kidney, ovary, prostate, uterus, } \\
\text { parathyroid, stomach }\end{array}$ \\
\hline stSG2362 & R38763 & Similarity to NAG-5 protein & & Brain, kidney, ovary, placenta \\
\hline stSG62716 & W42412 & No similarity & & Colon, heart, lung, ovary \\
\hline A003M47 & AF052116 & No similarity & & Brain \\
\hline T78312 & T78312 & No similarity & & Brain \\
\hline stSG52289 & AA634361 & No similarity & & Testis \\
\hline stSG55593 & D50406 & RECK (ST15) & $\begin{array}{l}\text { Membrane-anchored } \\
\text { glycoprotein (metastasis } \\
\text { and invasion) }\end{array}$ & $\begin{array}{l}\text { Brain, kidney, bone, heart, ovary, pancreas, } \\
\text { parathyroid, placenta, prostate, testis, uterus }\end{array}$ \\
\hline stSG39032 & Z46967 & Calicin (CCIN) & $\begin{array}{l}\text { Morphogenic cytoskeletal } \\
\text { element in spermiogenic } \\
\text { differentiation }\end{array}$ & Testis \\
\hline A002G23 & G26403 & No similarity & & $\begin{array}{l}\text { Blood, breast, brain, colon, heart, kidney, lung, } \\
\text { ovary, prostate, thymus, uterus, placenta, } \\
\text { lung, liver, skeletal muscle, pancreas }\end{array}$ \\
\hline M20471 & M20471 & Clathrin light chain A (CLTA) & $\begin{array}{l}\text { Regulatory element in } \\
\text { clathrin function }\end{array}$ & $\begin{array}{l}\text { Brain, breast, colon, heart, bone, kidney, lung, } \\
\text { lymph, skeletal muscle, ovary, pancreas, } \\
\text { parathyroid, placenta, prostate, spleen, testis, } \\
\text { stomach, liver, uterus }\end{array}$ \\
\hline SHGC-32868 & G27648 & $\begin{array}{l}\text { UDP-N-acetylglucosamine-2-epimerase } \\
\text { (GNE) }\end{array}$ & $\begin{array}{l}\text { Bi-functional enzyme in the } \\
\text { biosynthesis of } \\
\mathrm{N} \text {-acetylneuraminic acid } \\
\text { (NeuAc) }\end{array}$ & $\begin{array}{l}\text { Breast, colon, liver, lung, parathyroid, placenta, } \\
\text { prostate, uterus, brain, kidney, skeletal muscle, } \\
\text { heart, pancreas }\end{array}$ \\
\hline stSG52662 & N71485 & No similarity & & Foetal liver/spleen \\
\hline SHGC-33912 & G29562 & No similarity & & Foetal liver/spleen \\
\hline SHGC-104 & D11961 & No similarity & & $\begin{array}{l}\text { Brain, heart, kidney, lung, skeletal muscle, } \\
\text { parathyroid, placenta, prostate, thymus, } \\
\text { uterus, liver, pancreas }\end{array}$ \\
\hline AA254410 & AA254410 & No similarity & & Foetal liver. spleen \\
\hline T83948 & T83948 & No similarity & & Mouse spleen \\
\hline stSG22652 & R95130 & No similarity & & Foetal liver/spleen \\
\hline KIAA0175 & D79997 & $\begin{array}{l}\text { Similarity to } X \text {.laevis protein } \\
\text { kinase, a putative transmembrane } \\
\text { domain }\end{array}$ & & $\begin{array}{l}\text { Bone, colon, heart, skeletal muscle, stomach, } \\
\text { uterus, tonsil }\end{array}$ \\
\hline WI-7677 & M96944 & PAX5 & $\begin{array}{l}\text { B-cell specific transcription } \\
\text { factor }\end{array}$ & Blood, lung, testis, tonsil \\
\hline
\end{tabular}

in clathrin gene function known to be involved in several pathways of lysosomal proteolysis, in various cell types. Clathrin has also been implicated in the pathology of Alzheimer's disease and Pick's disease. Immunohistochemical stainings of muscle fibre from Japanese HIBM patients did not lead to definitive conclusions about differential expression of this protein between those and control muscle tissue. ${ }^{32}$ According to these data, the involvement of CLTA in HIBM cannot be ruled out at this point. The involvement of the three known additional genes located in the interval in the pathogenesis of HIBM seems less likely: mutations in the bifunctional enzyme GNE have been related to sialuria, a rare inborn error of metabolism characterised by cytoplasmic accumulation and increased urinary excretion of free neuraminic acid; ST15 has been recently recognized as a matrix protein regulated tumour 


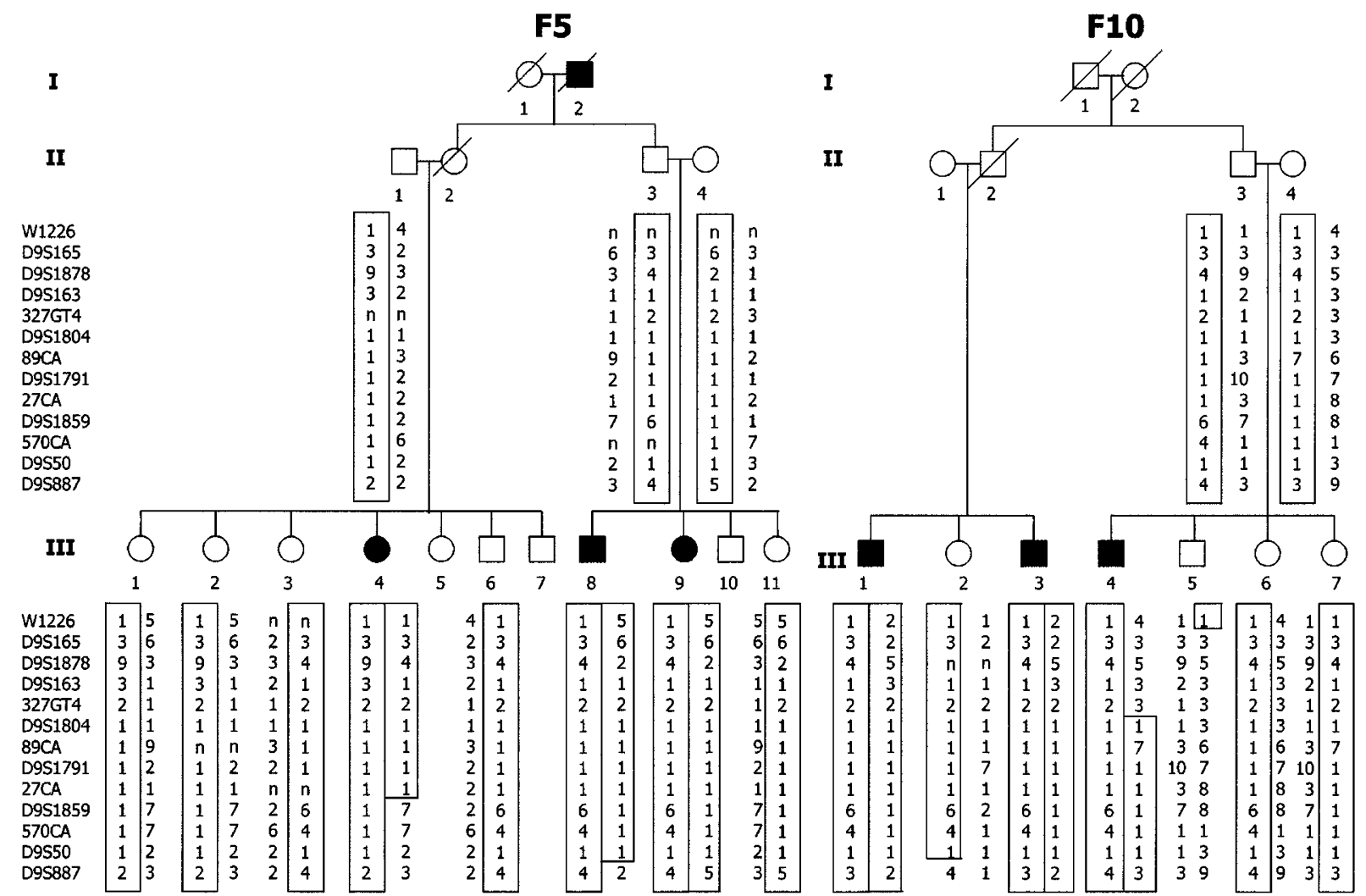

Figure 2 Haplotype analysis in two HIBM families showing recombination events in the vicinity of the HIBM locus. Haplotypes comprise alleles at the listed loci ordered telomeric to centromeric W1226, D9S165, D9S1878, D9S163, 327GT4, D9S1804, 89CA, D9S1791, 27CA, $D 951859,570 C A, D 9550$ and D9S887, ( $\mathrm{n}=$ not tested). Individuals are designated with generation and pedigree numbers. Disease status is indicated: affected, filled symbols; unaffected, clear symbols. Deceased individuals are denoted by slashes.

suppressor gene which can mediate a variety of signals to tumour invasion and metastasis. CCIN, a morphogenic cytoskeletal protein, is expressed solely in testis and related to differentiation.

Although none of those four genes has been directly involved in any known muscle function, their potential relevance or irrelevance to HIBM has still to be established.

\section{Discussion}

The positional cloning approach still represents a very potent tool for the identification of a disease gene of unknown biological or biochemical function. The first step towards the identification of genes located in a specific chromosomal fragment is the construction of a high resolution genomic map within this region.

Our previous studies localised the HIBM gene into a region of chromosome 9p12-p13 flanked by markers D9S1791 and D9S50 in a $1 \mathrm{Mb}$ interval laying on a single YAC. ${ }^{7,33}$ BAC end walking was used for the construction of a contig covering the relevant region. Analysis of single BAC libraries revealed the presence of gaps at different locations in the region. Only by screening three different BAC genomic libraries, we were able to establish a complete contig spanning the genes $\beta$ tropomyosin and the $3^{\prime}$ end of PAX5, which includes the HIBM candidate region. This continuous contig was used as the basis for the generation of a highly detailed physical and transcriptional map of the region. We have precisely ordered 40 different loci, and identified 13 new polymorphic markers which allowed the refinement of the haplotype analysis in HIBM families. Although four of the clusters could not be definitely ordered internally by BAC hybridisation, we have established their location from combined hybridisation results and data of all available databases relating to them (GeneMap99, The Sanger Center, GDB). Our previous genotyping studies with eight polymorphic markers located the HIBM disease in the interval flanked by D9S1791 and D9S50, based on both recombination and linkage disequilibrium data. The disease status in each subject was determined by personal clinical examination of one of us ( $\mathrm{Z}$ Argov and $\mathrm{M}$ Sadeh). Unfortunately, there are no known pre-symptomatic tests in HIBM to predict the status of a 'currently healthy' 
individual (like the markedly elevated serum creatine kinase before onset of muscle weakness in various muscular dystrophies). Since all of the patients in the HIBM families analysed were diagnosed before age 35 years, unaffected family members below the age of 35 years were excluded from analysis in our studies. The recombination event which located the distal boundary at D9S1791 was recognised in an unaffected individual which was examined at age 43 years and showed no sign of muscle disease then. Since in this family the age of onset was 33 years, that is 10 years before, the data observed pointed to a recombination event. However, a recent reexamination of the patient revealed the clinical criteria for HIBM diagnosis, so that we are now aware that some of the presently healthy members of the families studied may become symptomatic at a later time. Therefore only recombination events observed in affected individuals are taken into consideration. At present, we cannot consider D9S1791 as the distal boundary defining the HIBM interval; the closest unambiguous recombination event has been recognised at marker 327GT4 in an affected individual. Twenty-five genes and ESTs derived from all screened databases have been analysed for their precise location in the 9p12-p13 region, and for their expression pattern in a variety of tissues. Our results show that most of those transcripts are present in more than one tissue type, some are more specific to certain types, such as foetal liver or spleen, brain or testis. Furthermore no transcript was found to be expressed solely in skeletal muscle tissue. Among the 25 transcripts identified in the region, two cytoskeletal proteins, $\beta$-tropomyosin and talin, are the only genes with a recognised function in muscle, and therefore they were the most attractive genes to be potentially involved in HIBM. Both genes were subsequently excluded by position, by linkage disequilibrium $\operatorname{data}^{7}$ and, for talin, because a recombination event has been recognised with a polymorphic site within the gene ${ }^{24}$ in one affected individual. However, the function of many of the genes and ESTs mapped to 9p12-p13 has not yet been established, and the continuous flow of sequence and transcriptional data demands a constant update of our analysis, till the systematic sequencing of the entire interval is completed.

Our goal is the identification of all the genes in human chromosome 9p12-p13, including the HIBM interval laying at present between $327 T G 4$ and D9S1859. The high resolution physical and transcriptional map established in these studies will contribute to the positional cloning of various disease causing genes located in this region, among them the gene responsible for HIBM. Certainly the identification of the gene will have immediate implications such as efficient genetic counseling for HIBM families, and precise knowledge of all HIBM allelic disorders. Moreover, further characterisation of the HIBM causing gene will provide important insights into the mechanisms of inclusion body myopathies and increase our understanding of the molecular basis for impaired function of the muscle tissue.

\section{Acknowledgments}

We would like to thank Mira Korner and all the staff from The Laboratory of DNA Analysis at The Institute of Life Sciences, The Hebrew University of Jerusalem, for their skilful assistance in sequencing, and Anka Sanilevitch for her invaluable help in oligonucleotide synthesis. This study was supported by Hadasit (Medical Research Services Development Co. Ltd., a subsidiary for $R \& D$ of Hadassah Medical Organization), by Grant 96-00268 from the United States-Israel Binational Science Foundation (BSF), by a grant from the Association Francaise contre les Myopathies (AFM), and by a special donation from Hadassah Southern California, the Persian Group Council, Vanguard II, Healing Spirit and Haifa Group, and from the ARM organisation.

\section{References}

1 Argov Z, Yarom R. "Rimmed vacuole myopathy" sparing the quadriceps: a unique disorder in Iranian Jews. J Neurol Sci 1984; 64: $33-43$

2 Sadeh M, Gadoth M, Hadar H, Ben David E. Vacuolar myopathy sparing the quadriceps. Brain 1993; 16: 217-232.

3 Askanas V, Engel WK. New advances in the understanding of sporadic inclusion-body myositis and hereditary inclusionbody myopathies. Curr Opinion Rheumatology 1995; 7: 486-496.

4 Mitrani-Rosenbaum S, Argov Z , Blumenfeld A, Seidman CE, Seidman JG. Hereditary Inclusion Body Myopathy maps to chromosome 9p1-q1. Hum Mol Genet 1996; 5: 159-163.

5 Argov Z, Tiram E, Eisenberg I et al. Various types of hereditary inclusion body myopathies map to chromosome 9p1-q1. Ann Neurol 1997; 41: 548-551.

6 Argov Z, Sadeh M, Eisenberg I, Karpati G, Mitrani-Rosenbaum S. Facial weakness in hereditary inclusion body myopathies. Neurology 1998; 50: 1925 - 1926.

7 Eisenberg I, Thiel C, Levi T et al. Fine Structure Mapping of the Hereditary Inclusion Body Myopathy Locus. Genomics 1999; 55 $43-48$.

8 Christodoulou K, Papadopoulou E, Tsingis M et al. Narrowing of the gene locus for autosomal-recessive quadriceps sparing inclusion body myopathy (ARQS-IBM) to chromosome $9 \mathrm{p} 1$. Acta Myol 1998; 2: 7 -9.

9 Mirabella R, Christodoulou K, Di Giovanni S, Ricci E, Tonali P, Servidei S. An Italian family with autosomal recessive quadriceps-sparing inclusion-body myopathy (ARQS-IBM) linked to chromosome 9p1. Neurol Sci 2000; 21: 99-102.

10 Ikeuchi T, Asaka T, Saito M et al. Gene locus for autosomal recessive distal myopathy with rimmed vacuoles maps to chromosome 9. Ann Neurol 1997; 41: 432-437.

11 Sessarego M, Defferrari R, Fugazza G, Comelli A, Salvidio E, Ajmar F. Involvement of the short arm of the derivative chromosome 9 in Philadelphia- positive acute lymphoblastic leukemia. Cancer Genet Cytogenet 1991; 52: 43-49.

12 Gaidano G, Dalla-Favera R. Biologic and molecular characterization of non-Hodgkin's lymphoma. Curr Opinion Oncology 1993; 5: 776-784.

13 Kim SK, Ro JY, Kemp BL et al. Identification of three distinct tumor suppressor loci on the short arm of chromosome 9 in small cell lung cancer. Cancer Res 1997; 57: 400-403.

14 Liew CT, Li HM, Lo KW et al. Frequent allelic loss on chromosome 9 in hepatocellular carcinoma. Int J Cancer 1999; 81: $319-324$.

15 Bamshad M, Watkins WS, Zenger RK et al. A gene for distal arthrogryposis type I maps to the pericentromeric region of chromosome 9. Am J Hum Genet 1994; 55: 1153-1158.

16 Kant S, Polinkovsky A, Mundlos S et al. Acromesomelic dysplasia Maroteaux type maps to human chromosome 9. Am J Hum Genet 1998; 63: $155-162$. 
17 Sulisalo T, Sistonen P, Hastbacka J et al. Cartilage-hair hypoplasia gene assigned to chromosome 9 by linkage analysis. Nature Genetics 1993; 3: $338-341$.

18 Middleton LT, Christodoulou K, Mubaidin A et al. Distal hereditary motor neuropathy of the Jerash type. Ann NY Acad Sci 1999; 883: 439-442.

19 Deloukas P, Schuler GD, Gyapay G et al. A physical map of 30,000 human genes. Science 1998; 282: 744-746.

20 Altschul SF, Madden TL, Schaffer AA et al. Gapped BLAST and PSI-BLAST: a new generation of protein database search programs. Nucleic Acids Res 1997; 25: 3389-3402.

21 Widada JS, Ferraz C, Capony JP, Liautard JP. Complete nucleotide-sequence of the adult skeletal isoform of skeletal muscle beta-tropomyosin. Nucleic Acids Res 1988; 16: 3109.

22 Tiso N, Rampoldi L, Pallavicini A et al. Fine mapping of five human skeletal muscle genes: alpha-tropomyosin, beta-tropomyosin,troponin-I slow twitch, troponin-I fast twitch, and troponin-C fast. Biochem Biophys Res Commun 1997; 230: 347 350

23 Rees DJ, Ades SE, Singer SJ, Hynes RO. Sequence and domain structure of talin. Nature 1990; 347: 685-689.

24 Ben-Yosef T, Francomano CA. Characterization of the human talin (TLN) gene: genomic structure, chromosomal localization, and expression pattern. Genomics 1999; 62: 316-319.

$25 \mathrm{Lu}$ R, Yang P, O'Hare P, Misra V. Luman, a new member of the CREB/ATF family, binds to herpes simplex virus VP16-associated host cellular factor. Mol Cell Biol 1997; 17: 5117-5126.

26 Takahashi C, Sheng Z, Horan, TP et al. Inhibition of tumor invasion by the membrane-anchored glycoprotein RECK. Proc Natl Acad Sci USA 1998; 95: 13221 - 13226.
27 Hinderlich S, Stasche R, Zeitler R, Reutter W. A bifunctional enzyme catalyzes the first two steps in $\mathrm{N}$-acetylneuraminic acid biosynthesis of rat liver. Purification and characterization of UDP-N-acetylglucosamine 2-epimerase/N-acetylmannosamine kinase. I Biol Chem 1997; 272: 24313 -24318.

28 von Bulow M, Heid H, Hess H, Franke WW. Molecular nature of calicin, a major basic protein of the mammalian sperm head cytoskeleton. Exp Cell Res 1995; 219: 407 - 413.

29 Jackson AP, Parham P. Structure of human clathrin light chains. Conservation of light chain polymorphisms in three mammalian species. J Biol Chem 1988; 263: $16688-16695$.

30 Ponnambalam S, Jackson AP, LeBeau MM et al. Chromosomal location and some structural features of human clathrin lightchain genes (CLTA and CLTB). Genomics 1994; 24: 440-444.

31 Adams B, Dorfler P, Aguzzi A et al. Pax-5 encodes the transcription factor BSAP and is expressed in B lymphocytes, the developing CNS, and adult testis. Genes Dev 1992; 6: 15891607.

32 Kumamoto T, Abe T, Nagao S, Ueyama H, Tsuda T. Immunohistochemical study of clathrin in distal myopathy with rimmed vacuoles. Acta Neuropathol 1998; 95: 571-575.

33 Argov Z, Eisenberg I, Mitrani-Rosenbaum S. Genetics of inclusion body myopathies. Curr Opinion Rheumatology 1998; 10: $543-547$

34 Vakkilainen T, Kivipensas P, Kaitila I, de la Chapelle A, Ridanpaa $\mathrm{M}$. Integrated high-resolution BAC, P1, and transcript map of the $\mathrm{CHH}$ region in chromosome 9p13. Genomics 1999; 59: 319_ 325 . 